\title{
Solar System Architecture
}

\author{
Valentin Nikitovich Tkachev* \\ Moscow State University of Civil Engineering, Moscow, Russia \\ *Corresponding author.Email: valentintn@mail.ru
}

\begin{abstract}
The anthropic point of view has always been leading in understanding the universe, terrestrial and cosmic, in attempts to integrate cosmic phenomena with everyday experience, multiplying the impressions of the observed, but unattainable objects and Universe events by coefficients of greatness. Earthly architecture has indeed become a convincing reflection of the ambitious claims of the earthly lords to demonstrate kinship with the upper world. Hypothetical knowledge about the world was updated to the extent of concentric spheres expansion of penetration into cosmic depths, relying on scientific logic, supported by the interpretation of visual observation instrumental data. And today, "stitching" the data about the Universe laws into a single system of diverse manifestations of matter filling the cosmos, we are convinced that the structural parallels of earthly architecture with the construction of the cosmos are not the costs of the earthly, anthropic form of thinking, but an illustration of the natural metamorphoses of matter in the direction of improvement in the mode of birth, being, death - the implementation of space architecture. Although, we note that metaphors should not be orthodox and all-pervading, and we do not support the idea of the Universe birth. At the same time, the hypothetical logic of judgments about the birth and existence of the Solar System shows the mechanism of the Universe forces action in a small, "domestic" version. It seems today to be an impeccable equivalent of earthly architecture reality in the familiar concepts of tectonics, cyclical life, harmony, rhythm, expediency.
\end{abstract}

Keywords: Universe, regularities of matter metamorphosis, formation of galaxies, stars and planetary systems, Solar system, preconditions for organic life and mind

\section{INTRODUCTION}

Naive and daring intentions to reach out to Heaven, to reflect your understanding of the systemic nature of the world are captured in myths and legends about Hyperborea, the Tower of Babel and Mount Sumeru [1], the symbolism of the planning of ancient cities and grandiose architectural ensembles: Ecbatan, Angkor Vata, Chichen Itza [2 ]. (fig. 1.)

We observe all the space events only at a distance, and the distances from the Earth to any, even the nearest celestial body accessible to observation, are enormous. The universe models created speculatively were carried out, of course, in a compact version - diagrams and globes. The Earth is away from the Sun at a distance of half a light year, 150 million $\mathrm{km}$, from the Sun to Jupiter 600 million $\mathrm{km}$, to Pluto - 6 billion km. Sun light reaches it only after 20 light years.

Natural philosophers, who stood firmly on the ground and perceived it as the center of the world, did not understand its structure clearly [3].
Only Plato, who had a good fantasy, spatial imagination and a tendency towards romance, dared to depict a slender, albeit naive architecture hypothesis of "his" planetary community [4]. No wonder he is considered the father of idealism.

Evidence of his architectural thinking was a retelling of Atlantis layout, a planetary model showing the orbits of the known planets and the Sun revolving around the Earth, structure skatches of an ideal city, an attempt, following Pythagoras, to construct a cosmic building from mutually nested regular geometric bodies. Plato's fantasies about "singing spheres" and orbit sirens living on each planet impressed the astronomers most of all (Pic. 2.).

And two thousand years later, Kepler I., who calculated planets orbits, found that they are located in harmony with the laws of the music sequence [5].

Later, Hindemith P.,the German composer, voiced the ideas of planetary harmony in "Harmony of the World" symphonies [6]. The drama of replacing 


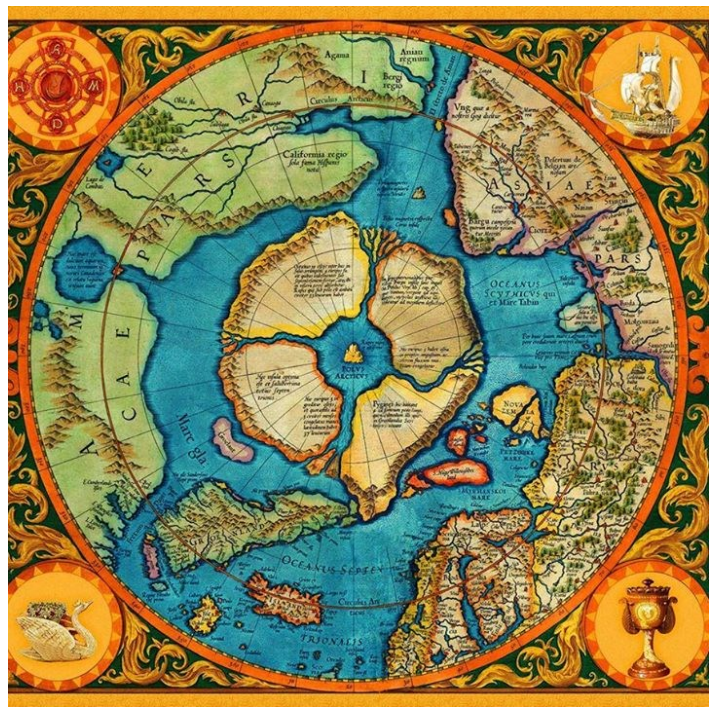

Figure 1. Medieval image of hypothetical Hyperborea.

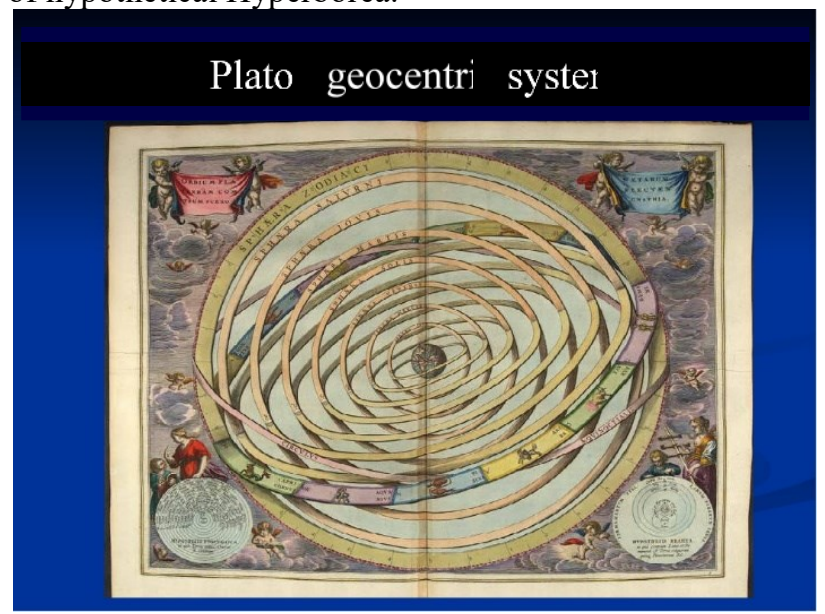

Figure 2 Ancient idea of the universe according to Plato

geocentrism with a heliocentric system ends beautifully on this musical note. Bruno G., Galilei G. and Copernicus $\mathrm{N}$. himself who noted that "it is time to open eyes to the real place of the Sun in its own planetary family" suffered for this idea in the struggle against the church. [7], (Pic. 3.).The problem of ordering the ideas about solar system structure has long ripened, and even the church was interested in establishing the coordination of planets movement and the calendar. Biblical events and religious holidays were linked to its dates.

The invention of the telescope and the rapid improvement of its capabilities opened up new depths of space and intensified the development of new hypotheses of the Universe structure with the same anthropic interpretation of what has been seen. People saw that obscure clumps of nebulae are giant clusters of stars organized into galaxies - vortex systems revolving around their centers. The gravitational theory of Newton I. has already become widespread in the explanation of celestial bodies formation principles and had a convincing development as the basic mechanism of the Universe life.

No worse than ancient natural philosophers, but only in a cosmic scale, astronomers and astrophysicists formed the understanding of the universe:

First, proceeding from the logic of the anthropic understanding that everything that exists should someday appear and then perish at the right time, the main idea was the following - the Universe was created out of nothing by the Big Bang. This idea finally reached an agreement between the religion and the science [8]...

How did it happen that the biblical myths were able to lead scientists away from the common sense that the universe - this infinite space - has existed and will continue to exist for all time? Is the Big Bang an episodic, repeated event of the matter metamorphosis, a consequence of the action of gravity on a cosmic scale?

Second, there is a fairly convincing (by earthly standards too!) Model of celestial bodies formation and 


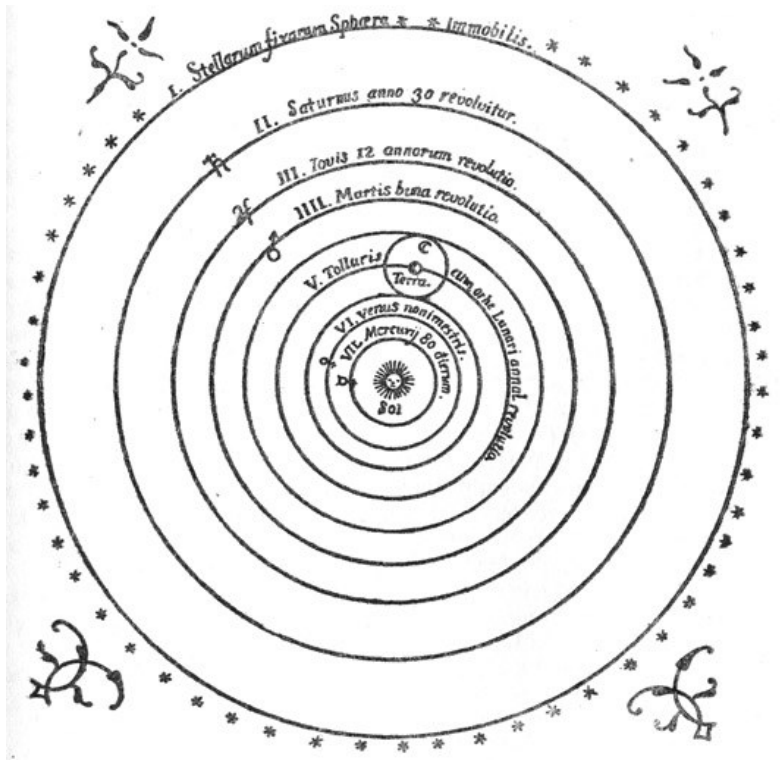

Figure 3 Copernicus N. heliocentric scheme.

their "behavior", described by Nikonov A. in the popular book "Riding a Bomb" [8]. It is difficult to convince yourself that the technology of matter metamorphosis in space has no beginning and always exists. You have to get used to it.

Stardust gathers into clouds, which, through the rotation and mutual matter gravity, are concentrated into spherical gas-dust protoplanets that do not have a hard shell, but due to the compaction and heating of the core by gravitational pressing, the "heavy" elements with atoms "hung" by a large number of electrons are formed in them. Their discharge causes a thermonuclear reaction and the star becomes radioactive.

Third, further "sticking" of matter attracted from space causes superdensification of the star, collapse and explosion. As a result, the fragments of the exploded mass are scattered throughout space in the form of asteroids or simply space debris.

This is the life of the Universe, dimensionless in space and time of existence.

It is a void where visible and invisible events of matter transformation, duplicated on a small scale by the Solar system, take place. This happens based on the Universe simple patterns.

\section{MATHERIALS ANS METHODS}

Note that according to the study of the universe events, a human with the rights of an earthly inhabitant and an observer is a point and a measure of reference for the phenomena of the macro- and microcosm of the cosmos.
Historically, the criteria for the adequacy of the construction of any structures were developed mainly for the sphere of the macrocosm, space.

The microcosm, perhaps for an unconscious inattention to its architecture, reminds of itself with pandemics. It should also be dealt with. On the other hand, information sources on the Universe - adequate and hypothetical in terms of reliability - have been abundant since antiquity. Moreover, the conjectures of natural philosophers of the 6th - 4th centuries BC. surprisingly accurate in anticipating modern findings in astrophysics and space philosophy. Of course, these findings are based on a positive interpretation of in-depth research "with a look" and a logic. It's reliability status is established by the interaction of several hypotheses in anthropic presentation.

It seems that Penrose $\mathrm{R}$. is right that modern cosmophysics is a poorly cut patchwork quilt [10]. But it is still impossible to establish the truth by touching the object with hands, especially since a person in his organic body cannot overcome the environmental rigidity beyond the alma mater of unimaginable distances in space, which have to be measured not by linear measures, but by the time of overcoming.

In order to see the distant stars a telescope had to be invented, thus in order to adequately convey the tactile sensations of an alien planet, it is necessary to invent clones with a physically indestructible body and an errorfree electronic brain. So, to avoid physical travel to distant worlds, the clone must be constructed by radio commands directly at the site of the distance point. 
We admit that somewhat ironic attitude to the hypotheses and providences of modern space physics allows to participate in comprehension and forecasting of the Universe events, relying more on archaic ideas about the world especially on the reflections of the ancient Indians about the universe fractal structure and Pythagorean ideas about the interconnection of the worlds.

We remain in the system of assessments developed by human earthly history in the filtration of archetypes of being understanding: the role of gravity, reflected in architectonics, rotation as a principle of creating forms (planets and pottery), movement in general as the basis of life in the matter cyclical existence. Universal patterns are brilliant in the results of action, but simple in nature.

On these assumptions made for ourselves, our further presentation of the topic is built, without fear of reproaches for amateurism.

\section{STUDY RESULTS}

The ideas of earthlings about their heavenly home periodically changed from the simplest naive in the form of a flat earth, covered with a crystal dome with fixed stars, to the awareness of themselves as citizens of the
Universe, which is not yet fully understood mechanism of spatial and temporal transformations of the matter filling it.

For overcoming the twilight ideas about the structure of the solar system, transferred to the universal scale of the stellar world, humanity paid with ostracism of the most astute scientists. They were leading people cognitive energy away from the geocentrism orthodox pictures to more realistic structures of "small space", where all the planets were set up in their places and harmony was achieved.

The victory of mastering the structural regularity of the nearest planetary system (and at the same time, by the way, the architecture of the periodic table of elements) paved a bridge to the identification of "non-random" observed events of the Universe, very reminiscent of the experience of terrestrial architecture.

The tectonics of terrestrial architecture is the historical result of gravity development, terrestrial gravity. Growing cities like predatory stars also attract the surrounding population and business, condense to a state of collapse and can also explode.

Both systems are characterized by signs of fractality [11] - everything is in everything - the centers of galaxies

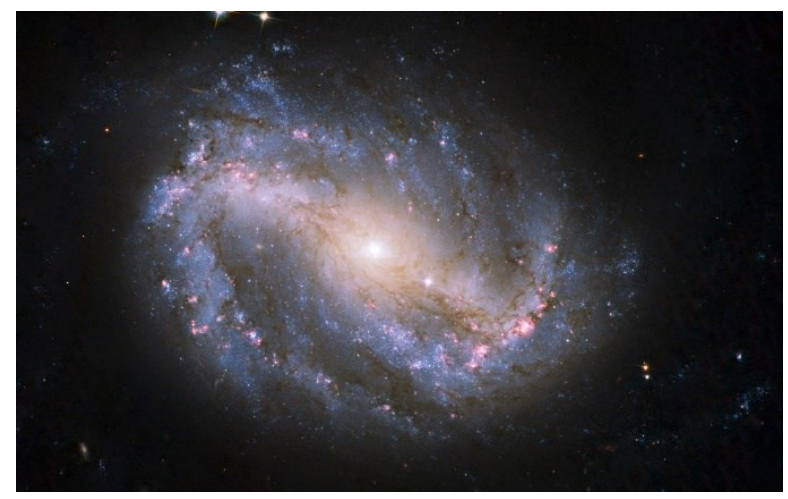

Figure 4. a) Formation of a spiral galaxy.

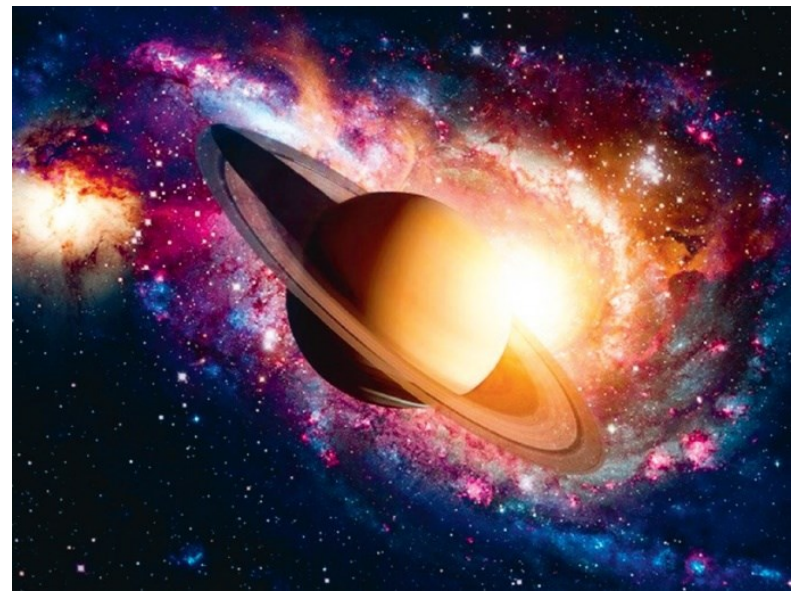

Figure 4. b) Saturn Planet, which has not completed the process of its formation. 
attract spiral trails of "their" planetary systems rotating around them; each holds its own satellites with a gravitational magnet, some of them, which are large, also have their own dust, gas, asteroids family like Saturn, (Pic. 4 a, b.).

Even Kant I., far from astrophysics, found the similarity of Saturn and its satellites ring with the ctructure of the solar system planets as a whole. He notoed that the massive density of planets decreases with their distance from the Sun [12]. Fractality in architecture is a method of formal harmonization of the facade and planning structure by repeating a geometric image [13].

Fractal patterns of structure are inherent - explicitly and implicitly - in many objects of the inert and living world. And, of course, it concerns matryoshkas as well. Now let's talk in more details about the structure of the solar system ensemble, as it appears today thanks to the results of observations that give the illusion of a fairly compact space unit like a globe museum model entangled in planetary orbits (in the Ptolemaic understanding).

The solar system is not an eternal universe. Supposedly it has been formed more than 4 billion years ago also from a dust and gas cloud, which, revolving around the formed center, the future Sun, in the plane of a single disk (this is also a regularity), was gradually coagulated by gravity, i.e. n. globules. According to Kant I. the density of their bodies increases in the planets close to the center.

A planetary hierarchy has formed: the most distant planets are dust and gas giants that have not yet had time to form into planets with a solid mass, like Mercury, Venus,

Earth, Mars, compacted to a small size. Rotating planets have one destiny - they will ultimately be swallowed up by the Sun; rotation only slows down this process (Pic. 5.).
The collective rotation of protoplanets in one orbital plane and in one direction excluded the possibility of internal collisions, decay; the variety of satellites was created by attracting the building material left over from the formation of planets. That is, the hypothetical destruction of Phaeton is unlikely.

The Sun is a moderate-sized star and swallowing its planets can add mass to it, resulting in a typical explosion of a collapsing star in the Universe. To complain about the limited reserves of hydrogen in the mass of the Sun, supposedly foreshadowing the depletion of fuel, the cooling and death of a star, means suspecting the Universe of finite reserves of chemical elements and the possibilities of their creation. Any finite quantities in the measurement of the Universe are meaningless, although the quantitative prerequisites for the formation of particular large and small planetary systems are determined by the volume of the initial dust and gas cloud.

The prediction of the death of the entire solar system in more than 12 billion years after birth is correct. And before this sad but recurring event, all the planets will gradually cover considerable distances attracted by the Sun. On the general distance scale, the Earth temporarily occupies the most favorable position from the biota formation point of view. Its orbit is in the zone of the socalled. "Green belt" [14], where the Sun heats, but does not burn and there is a sufficient amount of water, also successfully transformed into different states of aggregation. As a result, we have the opportunity to contemplate landscapes where water creates a blue sky and clouds, sea and snow mountain peaks. The water cycle on the planet, due to the temperature dynamics on the surface, gives rise to streams of fresh water and all living planet creatures are tuned to the its consumption.

The moderate speed of the Earth's rotation supports a soft change of heating and cooling as well as due to the tilt of the planet's axis of rotation to the orbital plane.

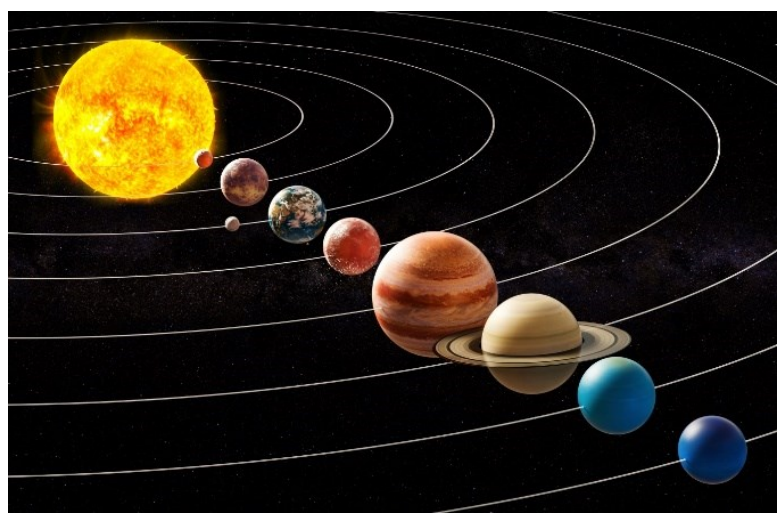

Figure 5 Schematic diagram of the solar system. 


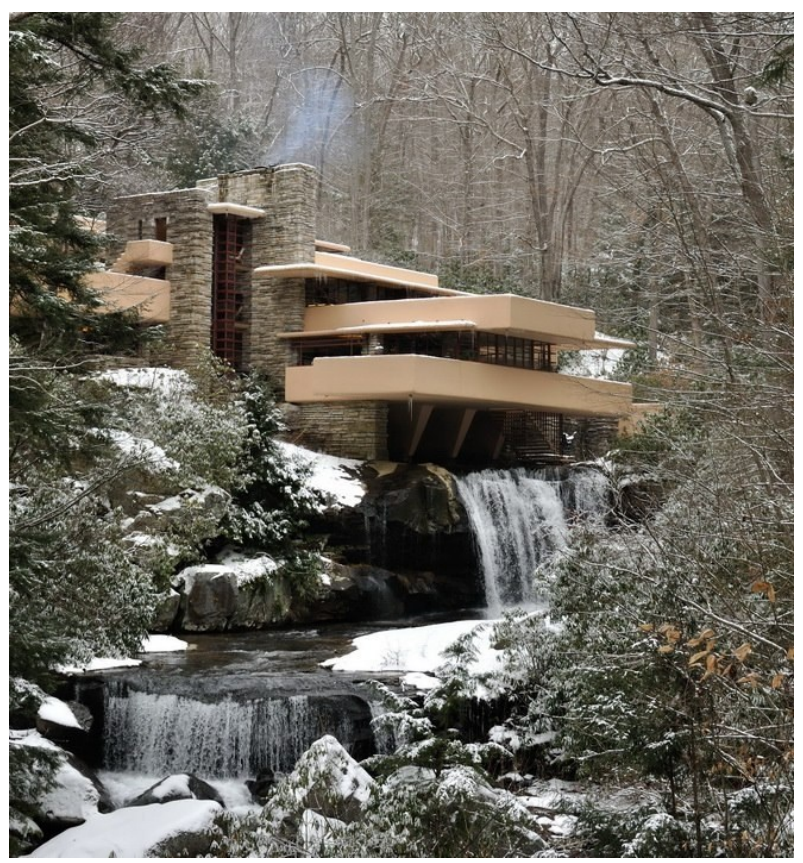

Figure 6 A house above a waterfall; arch. Wright F.-L.. The phenomenon of the implementation of Universe architectural laws on Earth.

Passing the path of rotation around the Sun, the Earth heats up the northern hemisphere for six months, the southern hemisphere for six months, and the change of seasons turned out to be a necessary condition for the adaptation of plants and animals ...

No matter how amazing the forms of living beings and their organic features are, they all naturally formed as a result of the change of millions individual forms generations. Their creator was an endless resource of time, a combination of the qualities of the external environment, supplemented by the lunar gravity pulsating effect, morphogenetic evolution - and no one else.

Everything that is born exists in this world temporarily.

There are systems in the Universe with the duration of life cycles which can create the illusion of their eternity. The subsystems filling them have foreseeable periods of existence from birth to death. These are planets, plants and animals species, including human races [15]. The sun with its planetary family is also a subsystem for its own galaxy, the Milky Way.

From time to time, episodic endoplanetary cataclysms (warming, glaciation, axis shift, pole change, etc.) cause evolutionary leaps that cancel out the achievements of the next morphogenetic experiment, and everything can start all over again. They believe the human race has its own lifetimes [16].
The rotation mechanism underlying the "globalization" of gas and dust clouds and the formation of planetary systems remains a mystery. This is a torsion mechanism, the role of which in cosmic morphogenesis is suspected, but a convincing hypothesis on this score does not yet exist, although that the principle of gravity in space is realized through rotation.

The formation of the solar system is not complete. All planets must pass the green belt and be drawn into the sun. New discoveries of miniplanets on the periphery of the system do not add anything significant to our existing ideas about the architecture of the solar planetary family in space and on Earth, harmoniously formed so that the impeccability of the image and outstanding structures of nature and man causes aesthetic awe (Pic. 6.).

\section{CONCLUSION}

1. Denial of key ideas about the Universe events and its birth from a point does not mean a lack of agreement on the matter morphogenesis basic issues according to the elementary laws of the Universe, not established by anyone, but logically following from the very essence of the life support patterns of cosmic processes. These include gravity and the dualism of electrical potentials, as well as the so-called "weak forces" explaining material transformations at the molecular level. Scientists lay these forces in the foundations of the cosmic theory of Everything.

2. The practice of the technical realization of these forces is based on the fractality mechanisms, i.e. 
arrangement of systems of similar elements, permeating all morphogenesis processes, starting from the universal scale and ending with miniature creatures of living and inert nature. The principle of fractality works as a tool for competent composition, where the general feature that characterizes the logic of morphogenesis and the ordering of forms is, of course, the reaction to the weight of the material used.

3. What is a purpose of this article which uses practically known information, including some author's deviations? This is an addition to the drawing of a line under another reached (albeit mostly hypothetically) stage of world comprehension as a manifestation of Universe uniform laws. The presentation of these patterns in terrestrial architecture emphasizes people insights on the commonality of terrestrial and celestial events in near space in the organization of the habitat since ancient times.

\section{REFERENCES}

[1] L.A. Waddell, The Buddhism of Tibet or Lamaism with its Mystic Cults, Symbolism and Mythology and its Relation to Indian Buddhism, in: Monasteries, Temples and Cathedrals (1958).

[2] J. Norman, Round cities, in: The World of Science, 12 (1983).

[3] N. Copernicus, On the circulation of the heavenly spheres (six books) (1964).

[4] A.F. Losev, History of antique aesthetics (1969).

[5] I. Kepler, About hexagonal snowflakes (1982).

[6] Yu.A. Danilov, Johannes Kepler and his "Harmony of the World", in: Sat. Patterns of symmetry (1980).

[7] V.N. Tkachev, The architecture of everything (2021).

[8] G. Smith, Mysticism and Science (an Intellectual Approach to Religion) (2017).

[9] A.P. Nikonov, Riding a bomb. The fate of the planet Earth and its inhabitants. https://www.litmir.me/br?b=103857\&p=1

[10] S. Hawking, Big, small and human mind (2014).

[11] B. Mandelbrot, Fractal geometry of nature (2002).

[12] I. Kant, Works in 6 volumes, 1 (1963).

[13] T.O. Sarvut, The experience of translating the mechanism of fractals theory to the development principles of Siberia and the Arctic habitat, in: Architecture and construction of Russia, 12 (2019).
[14] S. Hawking, Higher design (2018).

[15] S.D. Khaitun, Society against man. The laws of social evolution (2006).

[16] A.P. Nazaretyan, Nonlinear future (2017). 